\title{
Navigational Hypertext Models For Physical Hypermedia Environments
}

\author{
David E. Millard, David C. De Roure, \\ Danius T. Michaelides, \\ Mark K. Thompson, Mark J.'Weal, \\ University of Southampton, UK \\ \{dem, dder, dtm, mkt, mjw\} \\ @ecs.soton.ac.uk
}

\begin{abstract}
In this paper we identify a common aim between ubiquitous computing and hypertext systems: the desire to present navigable, located and structured information. We propose that existing navigational hypertext models might be valuable as a formalisation of ubiquitous information and explore the challenges of applying standard hypertext operations, such as anchor resolution, display and link traversal, to links that have physical anchors.
\end{abstract}

Categories and Subject Descriptors: H.1 [Information Systems]: Models and Principles

General Terms: Theory

\section{INTRODUCTION}

The use of situated context, placing users or artifacts in locations and using that placement as a primary data source, has been evident in a number of information systems in ubiquitous computing environments [7], which implicitly link location context to applications or information spaces.

Explicit linking has long been the subject of hypermedia research. In particular, Open and Adaptive Hypermedia aim to create systems that are aware of a readers preferences or navigational choices and can guide them appropriately with contextualised links and content.

The term Physical Hypermedia has been used to describe any hypermedia system that deals with properties of the physical world $[1,6]$. In this paper we examine how existing navigational models and link structures (such as OHP or FOHM [5]) might cope with mixed reality scenarios.

\section{PHYSICAL HYPERMEDIA}

Our observations were made during our participation in the Mack Room experiment of the Equator City project [3]. We realised that while navigational hypermedia seems a

Permission to make digital or hard copies of all or part of this work for personal or classroom use is granted without fee provided that copies are not made or distributed for profit or commercial advantage and that copies bear this notice and the full citation on the first page. To copy otherwise, to republish, to post on servers or to redistribute to lists, requires prior specific permission and/or a fee.

HT'04, August 9-13, 2004, Santa Cruz, California, USA.

Copyright 2004 ACM 1-58113-848-2/04/0008 ...\$5.00. good fit to the problems of information provision in ubiquitous environments, there are differences between hypermedia operations on the desktop and those that can occur in mixed reality.

\subsection{Resolution of Anchors}

In traditional hypermedia links, the anchor contains information that specifies the source object, often in explicit terms, for example a URI. When links are permitted to have physical anchors, their anchor resolution can become much more complex. For example: location systems could be used to detect a person co-located with a source anchor; an explicit activation interface (such as a barcode) might in the physical location of the anchor to be activated (scanned); or the environment of a user could be implicitly monitored and pattern matched for symbolic markers.

\subsection{Display of Anchors}

Within physical environments anchors can be indicated via some form of sign. Signs might be digitally addressable, such as screens for visual display or speakers for sonification. An alternative strategy is to provide a digital representation of the physical world (such as a map), into which physical anchors can be digitally placed. Virtual or Augmented Reality could also be used. Issues of 'link overload' are especially pertinent in physical spaces, as the notification of an anchor's presence diverts a user's task focus. An explicit query-oriented modality thus appears more appealing than universal display, unless the anchors are guaranteed to be of high quality (e.g. personalised).

\subsection{Link Traversal}

When a link exists in the digital realm, the idea of traversal is often quite transparent to the user, but in the physical realm, the onus can be on the user to perform the traversal. E.g., we might have a link from a painting in a gallery to another painting in the same gallery: once the system has resolved the source anchor it presents the link to the user (which may involve presenting a thumbnail of the destination if appropriate), but to follow the link the user has to move to the location of the destination painting themselves.

\section{DISCUSSION}

In the Mack Room experiments we attempted to apply an existing OHS, the Auld Linky link server [4], across both digital and physical spaces. Anchor Resolution was achieved by 
using an ultrasonic location system to co-locate users with anchor regions, Anchor Display was achieved using a digital map on a hand-held device, but Link Traversal required us to re-evaluate our link structures, and somehow model additional traversal information. The FOHM [5] link structure shown in Figure 1 shows how we achieved this. In addition to the source and destination anchors attached to the link object, three pieces of additional information are attached:

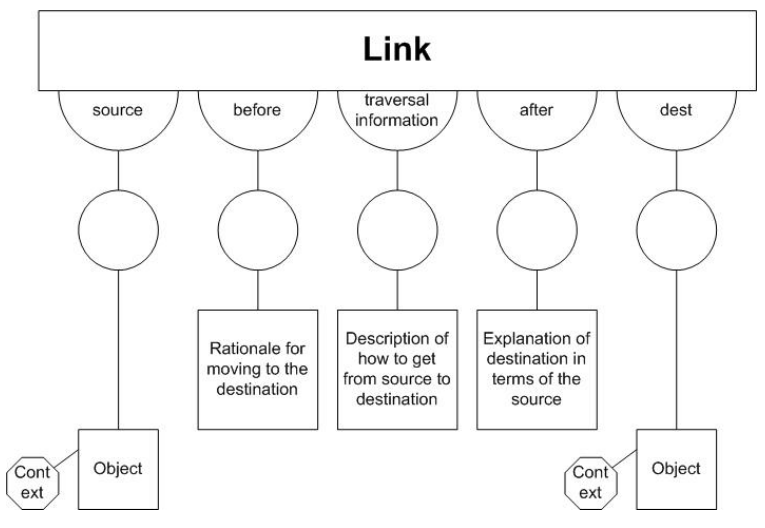

Figure 1: Mack Room Link Structure

Before: Provides a rationale for why the visitor may wish to move from the source to the destination. E.g. "A better example of Seurat's pointilist style can be seen in 'Sunday at the Grande Jatte.'"

Traversal Information: Describes how the visitor gets from the source to the destination. This could be map based or pre-authored instructions. E.g. 'Exit through the door at the end then turn left.'

After: Describes the destination in the context of having previously been at the source. E.g. 'As you can now see, unlike in the Bathers, Seurat has used much finer brushstrokes.' (This binding was included to simplify authoring, an alternative approach would be to use context/behaviour mechanisms to modify the destination if the viewer had previously viewed the source.)

Traversal support is an important part of this structure. Digital to digital traversal is typically swift, so hypermedia systems tend to offer their users little information on the traversal before it is taken. A common exception is to use some form of destination preview (E.g., hijacking the 'ALT' attribute within HTML). Open Hypermedia navigational links typically contain a description field that can be used in the same way, however the semantics of the description field are weak and its format prescribed (in OHP and FOHM it must always be a text string) and exceptional to the functioning of the hypermedia system (i.e. it cannot be a separate structure, such as a virtual document). This has implications for contextual or adaptive systems based on such models, as they cannot exercise the same degree of control over link metadata as they can over link members.

We have also observed that there is a difference in what OHSs and Ubiquitous Systems consider as context and what they include in their more structured information. Location in most ubiquitous systems is included in the context information, and can be somewhat indeterminate (as different sensors can report different locations for the same user position). In contrast, hypermedia systems model location much more explicitly in the form of anchors.

There is an historical connection between the two in that early work on link models often described anchors as the context of link endpoints [2]. It seems that later work on hypermedia models naturally incorporated anchors into the link models themselves and when context was then more generally addressed in adaptive and contextual hypermedia systems the anchors remained specialised.

In physical hypermedia we find a possible challenge to this view that states that in a contextual hypermedia system the anchors should remain part of the user's context, because not only is this more consistent with the rest of the context modelling but it also makes it possible to define anchor resolution in terms of context matching rather than embedding it in the hypermedia viewers. But this greater flexibility results in sophistication and higher complexity, which might exaggerate authoring problems that already exist.

We are currently taking these observations forward in a new physical hypermedia deployment, where we are combining Auld Linky with a recommender system. Unlike purely digital traversals, the user's potential investment in a physical link is large (involving considerable effort to reach the destination), and it is our hope that a recommender system will help to increase the quality of the links offered.

In this paper we have outlined new challenges for navigational hypermedia in physical spaces, from this new perspective we have given a short critique of existing traditional link models, and suggested new requirements for future physical navigational link structures.

\section{ACKNOWLEDGMENTS}

This research is funded by EPSRC IRC project "EQUATOR" GR/N15986/01.

\section{REFERENCES}

[1] K. Grønbæk, J. F. Kristensen, P. Ørbæk, and M. A. Eriksen. Physical hypermedia: Organising collections of mixed physical and digital material. In $A C M$ Hypertext'03, Nottingham, pages 10-19, 2003.

[2] L. Hardman, D. C. Bulterman, and G. van Russum. Links in Hypermedia: the Requirement for Context. In ACM Hypertext'93, Seattle, WA, pages 183-191, 1993.

[3] I. MacColl, B. Brown, S. Benford, and M. C. et al. Shared Visiting in Equator City. In $A C M C V E^{\prime} 02$, Bonn, pages 88-94, 2002.

[4] D. T. Michaelides, D. E. Millard, M. J. Weal, and D. C. De Roure. Auld leaky: A contextual open hypermedia link server. In Proceedings of $\mathrm{OHS}-7, \mathrm{SC}-3$ and AH-3, Published in LNCS 2266, pages 59-70, 2001.

[5] D. Millard, L. Moreau, H. Davis, and S. Reich. FOHM: A Fundamental Open Hypertext Model for Investigating Interoperability Between Hypertext Domains. In ACM Hypertext'00, San Antonio, TX, pages $93-102,2000$.

[6] L. Romero and N. Correia. Hyperreal: a hypermedia model for mixed reality. In ACM Hypertext'03, Nottingham, pages 2-9. ACM Press, 2003.

[7] J. Scholtz. Ubiquitous computing goes mobile. Mobile Computing and Communications Review, 5(3):32-8, July 2001. 